\title{
Integration of second order linear differential equations in elementary functions, example from quantum-mechanics
}

\author{
Alexander Chichurin ${ }^{1, *}$ and Viktor Red'kov ${ }^{2}$ \\ ${ }^{1}$ The John Paul II Catholic University of Lublin, Institute of Mathematics and Computer Science, ul. \\ Konstantynów 1H, Lublin, 20-708 \\ ${ }^{2}$ B. I. Stepanov Institute of Physics, NAS of Belarus, 68-2 Prospekt Nezavisimosti, Minsk 220072, \\ Belarus
}

\begin{abstract}
In the paper, special procedure for integrating a second order linear differential equation with six singular points, example of a quantummechanical problem for a spin zero particle with intrinsic Darwin-Cox structure, is considered. The method is based on the use of the Schwarzian derivative, conditions for parameters are derived at which solutions of the equation under consideration are constructed in terms of elementary functions. The graphs for several solutions are presented, their physical sense is discussed.
\end{abstract}

\section{Introduction}

In [1-3], a special method for constructing solutions for second order equations

$$
y^{\prime \prime}+p(x) y^{\prime}+q(x) y=0,
$$

where $p(x)$ is smooth function, $q(x)$ is continuous function. Let us assume that we know a particular solution $y_{1}(x)$ with given initial data $x=x_{0}, y\left(x_{0}\right)=y_{0}, y^{\prime}\left(x_{0}\right)=y_{0}$. Let other linearly independent solution be presented as $y=\xi(x) y_{1}$, where $\xi(x)$ stands for some unknown function. Differentiating twice the last relation, we get two equations

$$
2 \xi^{\prime} y_{1}+\left(p \xi^{\prime}+\xi^{\prime \prime}\right) y_{1}=0, \quad\left(3 \xi^{\prime \prime}-p \xi^{\prime}\right) y_{1}+\left(p \xi^{\prime \prime}+p^{\prime} \xi^{\prime}-2 q \xi^{\prime}+\xi^{\prime \prime \prime}\right) y_{1}=0 .
$$

This system is resolvable if the function $\xi^{\prime}(x)$ obeys the Schwartz equation

$$
2 \xi^{\prime} \xi^{\prime \prime \prime}-3 \xi^{\prime \prime 2}+\left(p^{2}+2 p^{\prime}-4 q\right) \xi^{\prime 2}=0 .
$$

Setting $\xi^{\prime}(x)=\eta(x), \eta^{\prime}(x)=w(x) \eta(x)$, for function $w(x)$ we derive the Riccati equation

$$
2 w^{\prime}=w^{2}-\left(p^{2}+2 p^{\prime}-4 q\right) \text {. }
$$

Thus, for finding general solution of eq. (1) it suffices to know a particular solution of Riccati (4). Note that within such an approach, we do not need to know in advance the partial solution $y_{1}$. Let us make the substitution $w(x)=v(x)-p(x)$, then eq. (4) takes the

\footnotetext{
* Corresponding author: achichurin@gmail.com
} 
form

$2 v^{\prime}=v^{2}-4 p v+4 q$.

On extension of this approach for linear differential equation of 3,4,5-th order see in [4]-[6].

\section{Results}

We will apply this method to the following differential equation $[7,8]$

$$
\left[\frac{d^{2}}{d x^{2}}+\left(\frac{1 / 2}{x-1}+\frac{1 / 2}{x}\right) \frac{d}{d x}-\frac{A}{x-1}-\frac{B}{x}-\frac{C}{x-(1-\gamma)}-\frac{D}{x-(1+\gamma)}\right] y=0,
$$

where

$$
A=\frac{\beta}{4 \gamma}+\frac{\varepsilon+1}{4}, B=\frac{\beta \gamma+\Lambda}{4\left(1-\gamma^{2}\right)}+\frac{1}{4}(-\varepsilon-1), C=-\frac{\beta+\Lambda}{8(1-\gamma) \gamma}, D=\frac{\Lambda-\beta}{8 \gamma(\gamma+1)} .
$$

Parameter $\varepsilon>0$ stands for energy; quantization of parameter $\Lambda>0$ is known from the analysis of the equation in the transversal coordinate; the parameter $\gamma$ is associated with an additional intrinsic structure, it is assumed to be sufficiently small; parameter $\beta>0$ means curvature of the space. This task arises when studying quantum-mechanical problem for a spin zero particle with intrinsic Darwin-Cox structure in presence of external magnetic field on the background of 3-dimensional spherical Riemann space [7, 8].

We will search solutions for corresponding equation (6) in the following form

$$
v=\frac{\sum_{i=0}^{4} d_{i} x^{4-i}}{x(x-1)^{2}(x-\gamma-1)(x-\gamma+1)},
$$

where $d_{i}(i=\overline{0,4})$ are unknown coefficients. We assume that $\gamma \neq-1,0,1$; also the notation $\varepsilon=\tau^{2}-1, \quad-1<\tau<1$ will be used. Substituting (7) in eq. (6), we obtain algebraic equation of 8 -th degree of the variable $x$, whence follows the system of 9 equations. Two equations from these 9 ones determine possible values for $d_{0}$ and $d_{4}$ :

$$
\mathrm{I}, \quad d_{0}=-\tau ; \quad \mathrm{II}, d_{0}=\tau ; \quad \mathrm{III}, d_{4}=0 ; \quad \mathrm{IV}, d_{4}=\gamma^{2}-1 .
$$

First, we consider the variant I, III. In this case, solutions of eq. (7) exist if the simple algebraic equation is satisfied:

$$
(\beta-\Lambda-4)(\beta-\Lambda)(\beta+\Lambda)(\beta+\Lambda+4)\left(\Lambda-\tau^{2}-\tau\right)=0
$$

From vanishing the first multiplier in (9) follows $\tau=-1(\varepsilon=0)$, however the case is of no interest in physical reason.

Let us consider the second multiplier in (9), this gives

$$
\Lambda=\beta, \quad \beta=2(1-\gamma), \quad \tau=2(\varepsilon=3) .
$$

At this, eq. (6) takes more simple form

$$
y^{\prime \prime}+\frac{2 x-1}{2(x-1) x} y^{\prime}-\frac{2 x-1}{2(x-1) x(\gamma+x-1)} y=0 .
$$

Its linearly independent solutions are (the case 1)

$$
\begin{gathered}
y_{1}=\gamma+x-1, \\
y_{2}=(\gamma+x-1)\left(\frac{(2 \gamma-1) \tanh ^{-1}\left(\frac{1}{\sqrt{\frac{y-1}{\gamma}} \sqrt{\frac{x-1}{x}}}\right)}{(\gamma-1)^{3 / 2} \gamma^{3 / 2}}-\frac{\sqrt{x-1} \sqrt{x}}{(\gamma-1) \gamma(\gamma+x-1)}\right) .
\end{gathered}
$$

The function $y_{2}$ is illustrated by fig. 1 (values for parameters are: $\varepsilon=3, \beta=10, \gamma=-2, \Lambda=10$ ) 

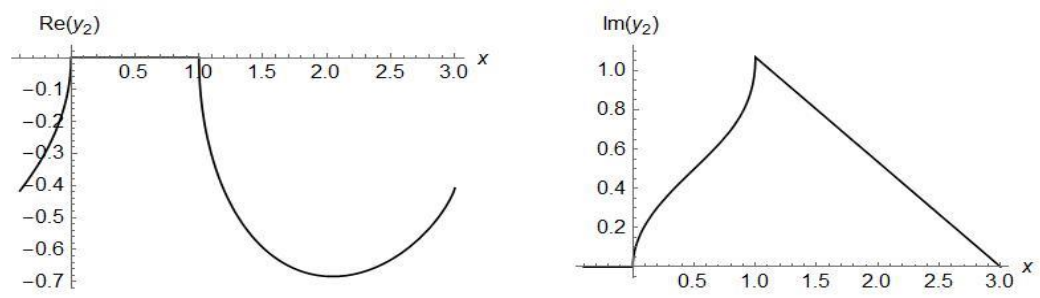

Fig. 1. Graphs of the real and imaginary parts of function $y_{2}(x)$.

Below we write down only final results.

\section{The case 2.}

$$
\begin{gathered}
\Lambda=\beta, \beta=2(3-4 \gamma), \tau=3 ; \\
y^{\prime \prime}+\frac{2 x-1}{2(x-1) x} y^{\prime}+\frac{3-\gamma-9 x}{4(x-1) x(\gamma+x-1)} y=0 ; \\
y_{1}=\sqrt{x-1}(\gamma+x-1), \\
y_{2}=\sqrt{x-1}(\gamma+x-1)\left(\frac{(3-4 \gamma) \tanh ^{-1}\left(\frac{1}{\sqrt{\frac{\gamma-1}{\gamma}} \sqrt{\frac{x-1}{x}}}\right)}{(\gamma-1)^{3 / 2} \gamma^{5 / 2}}+\frac{\frac{x-1}{(\gamma-1)(\gamma+x-1)}-2}{\gamma^{2} \sqrt{\frac{x-1}{x}}}\right) .
\end{gathered}
$$

The function $y_{2}$ is illustrated by fig. 2 (values for parameters are: $\varepsilon=8, \beta=22, \gamma=-2, \Lambda=22$ ).
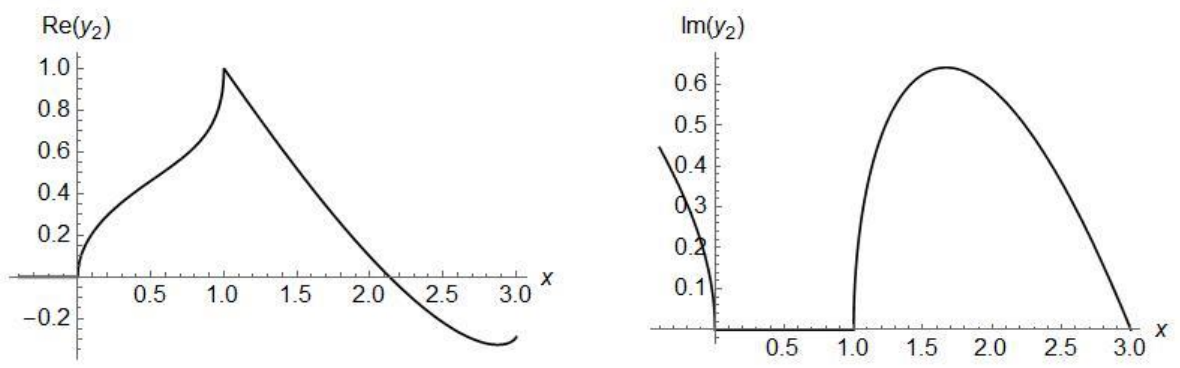

Fig. 2. Graphs of the real and imaginary parts of function $y_{2}(x)$.

\section{The case 3 .}

$$
\begin{gathered}
\Lambda=-\beta, \beta=-2(1+2 \gamma), \tau=2 ; \\
y^{\prime \prime}+\frac{2 x-1}{2(x-1) x} y^{\prime}-\frac{2 x-1}{2(x-1) x(x-\gamma-1)} y=0 ; \\
y_{1}=x-\gamma-1, \\
y_{2}=(x-\gamma-1)\left(\frac{(2 \gamma+1) \tanh ^{-1}\left(\frac{\sqrt{\frac{x-1}{x}}}{\sqrt{\frac{\gamma}{\gamma+1}}}\right)}{\gamma^{3 / 2}(\gamma+1)^{3 / 2}}+\frac{\sqrt{x-1} \sqrt{x}}{\left(\gamma^{2}+\gamma\right)(\gamma-x+1)}\right) .
\end{gathered}
$$




\section{The case 4.}

$$
\begin{gathered}
\Lambda=-\beta, \beta=-2(3+4 \gamma), \tau=3 \\
y^{\prime \prime}+\frac{2 x-1}{2(x-1) x} y^{\prime}+\frac{\gamma-9 x+3}{4(x-1) x(x-\gamma-1)} y=0 ; \\
y_{1}=\sqrt{x-1}(x-\gamma-1), \\
y_{2}=\sqrt{x-1}(x-\gamma-1)\left(\frac{(4 \gamma+3) \tanh ^{-1}\left(\frac{\sqrt{\frac{x-1}{x}}}{\sqrt{\frac{\gamma}{\gamma+1}}}\right)}{\gamma^{5 / 2}(\gamma+1)^{3 / 2}}+\frac{\frac{1-x}{(\gamma+1)(-\gamma+x-1)}-2}{\gamma^{2} \sqrt{\frac{x-1}{x}}}\right) .
\end{gathered}
$$

The case 5 .

$$
\begin{gathered}
\Lambda=\tau(\tau-1), \beta=-16 \gamma, \tau=4 ; \\
y^{\prime \prime}+\frac{2 x-1}{2(x-1) x} y^{\prime}+\frac{1-4 x}{x\left((x-1)^{2}-\gamma^{2}\right)} y=0 ; \\
y_{1}=(x-1)^{2}-\gamma^{2}, \\
y_{2}=\frac{\left((x-1)^{2}-\gamma^{2}\right)}{4 \gamma^{7 / 2}}\left(-\frac{2 \gamma^{3 / 2}(x-2) \sqrt{x-1} \sqrt{x}}{(\gamma-1)(\gamma+1)(x-\gamma-1)(\gamma+x-1)}+\right. \\
\left.\frac{(4 \gamma+3) \tan ^{-1}\left(\frac{\sqrt{\gamma} \sqrt{x}}{\sqrt{-\gamma-1} \sqrt{x-1}}\right)}{(-\gamma-1)^{3 / 2}}+\frac{(4 \gamma-3) \tanh ^{-1}\left(\frac{\sqrt{\gamma} \sqrt{x}}{\sqrt{\gamma-1} \sqrt{x-1}}\right)}{(\gamma-1)^{3 / 2}}\right) .
\end{gathered}
$$

The case 6.

$$
\begin{gathered}
\Lambda=\tau(\tau-1), \beta=-24 \gamma, \tau=5 ; \\
y^{\prime \prime}+\frac{2 x-1}{2(x-1) x} y^{\prime}+\frac{\gamma^{2}+5(6-5 x) x-5}{4(x-1) x(x-\gamma-1)(\gamma+x-1)} y=0 ; \\
y_{1}=\sqrt{x-1}\left((x-1)^{2}-\gamma^{2}\right), \\
y_{2}=\frac{1}{4(\gamma-1) \gamma^{9 / 2}} \times\left(\frac{(1-\gamma)(6 \gamma+5) \sqrt{x-1}\left((x-1)^{2}-\gamma^{2}\right) \tan ^{-1}\left(\frac{\sqrt{\gamma}}{\sqrt{-\gamma-1} \sqrt{\frac{x-1}{x}}}\right)}{(-\gamma-1)^{3 / 2}}+\right. \\
\frac{(6 \gamma-5) \sqrt{x-1}\left((x-1)^{2}-\gamma^{2}\right) \tanh ^{-1}\left(\frac{1}{\sqrt{\frac{\gamma-1}{\gamma}} \sqrt{\frac{x-1}{x}}}\right)}{\sqrt{\gamma-1}}+ \\
\left.\frac{2 \sqrt{\gamma} \sqrt{x}\left(-4 \gamma^{4}+\gamma^{2}(x(4 x-7)+7)-5(x-1)^{2}\right)}{\gamma+1}\right) .
\end{gathered}
$$

The function $y_{2}$ is illustrated by fig. 3 (values for parameters are: $\varepsilon=24, \beta=48, \gamma=-2$, $\Lambda=20)$. 

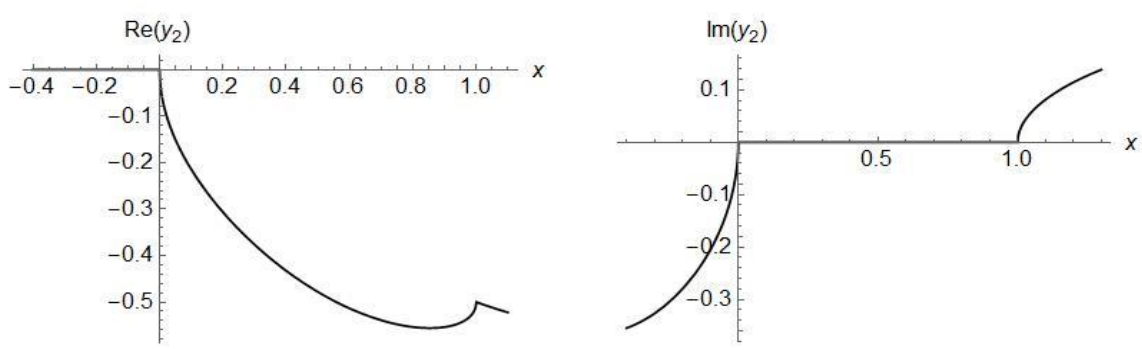

Fig. 3. Graphs of the real and imaginary parts of function $y_{2}(x)$.

Now we consider the variant I, IV.

\section{The case 7.}

$$
\begin{gathered}
\Lambda=\beta, \beta=2(1-4 \gamma), \tau=3 \\
y^{\prime \prime}+\frac{2 x-1}{2(x-1) x} y^{\prime}+\frac{7-\gamma-9 x}{4(x-1) x(\gamma+x-1)} y=0 \\
y_{1}=-\sqrt{x}(\gamma+x-1) \\
y_{2}=-\sqrt{x}(\gamma+x-1)\left(\frac{(1-4 \gamma) \tanh ^{-1}\left(\frac{1}{\sqrt{\frac{\gamma-1}{\gamma}} \sqrt{\frac{x-1}{x}}}\right)}{(\gamma-1)^{5 / 2} \gamma^{3 / 2}}+\frac{\sqrt{\frac{x-1}{x}}(2(\gamma-1) \gamma+2 \gamma x+x)}{(\gamma-1)^{2} \gamma(\gamma+x-1)}\right)
\end{gathered}
$$

Functions $y_{1}$ and $y_{2}$ are illustrated by fig. 4, 5 (parameters: $\varepsilon=8, \beta=18, \gamma=-2, \Lambda=18$ ).

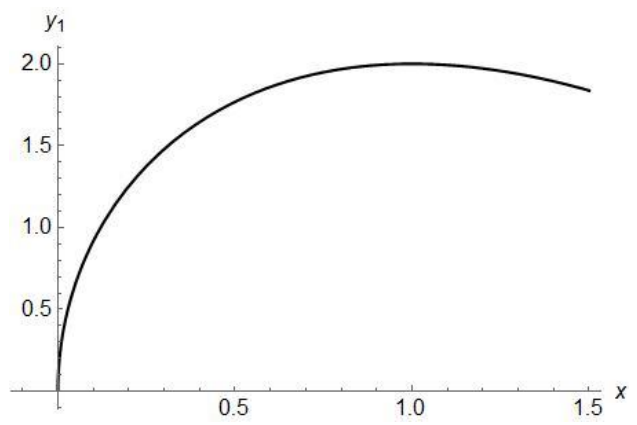

Fig. 4. The graph of the function $y_{1}(x)$.
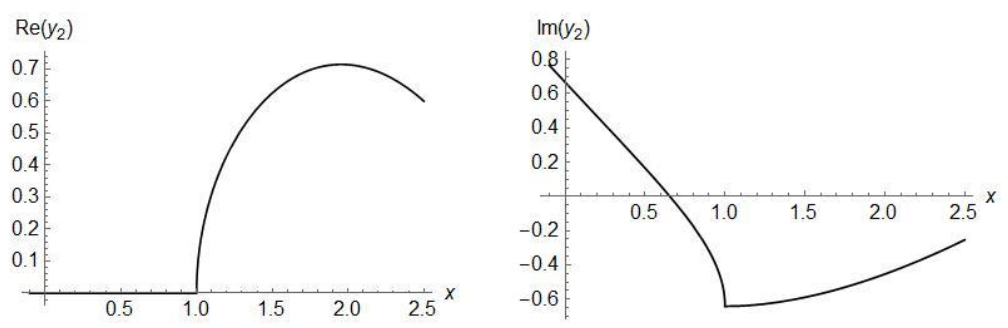

Fig. 5. Graphs of the real and imaginary parts of function $y_{2}(x)$. 
The case 8 .

$$
\begin{aligned}
& \Lambda=\beta, \beta=6(1-2 \gamma), \tau=4 ; \\
& y^{\prime \prime}+\frac{2 x-1}{2(x-1) x} y^{\prime}+\frac{5-2 \gamma-8 x}{2(x-1) x(\gamma+x-1)} y=0 \text {; } \\
& y_{1}=-\sqrt{(1-x) x}(\gamma+x-1) \text {, } \\
& y_{2}=\sqrt{-(x-1) x}\left(( \gamma + x - 1 ) \left(\frac{3(2 \gamma-1) \tanh ^{-1}\left(\frac{1}{\sqrt{\frac{\gamma-1}{\gamma}} \sqrt{\frac{x-1}{x}}}\right)}{(\gamma-1)^{5 / 2} \gamma^{5 / 2}}-\right.\right. \\
& \left.\sqrt{\frac{x-1}{x}}\left(\frac{2}{(\gamma-1)^{2}}+\frac{x}{\gamma^{2}}\left(\frac{1}{(\gamma-1)^{2}(\gamma+x-1)}+\frac{2}{x-1}\right)\right)\right) .
\end{aligned}
$$

Solutions $y_{1}$ and $y_{2}$ are illustrated by fig. 6, 7 (parameters: $\varepsilon=15, \beta=30, \gamma=-2, \Lambda=30$ ).

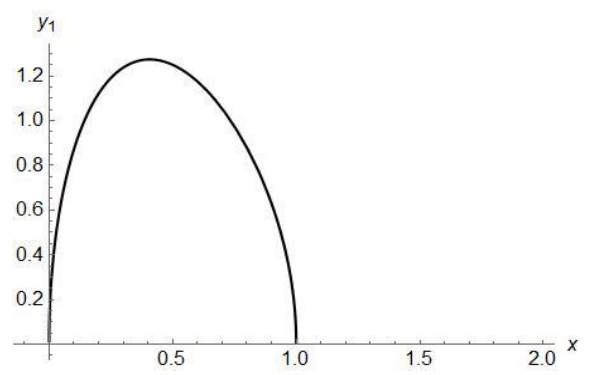

Fig. 6. The graph of the function $y_{1}(x)$.
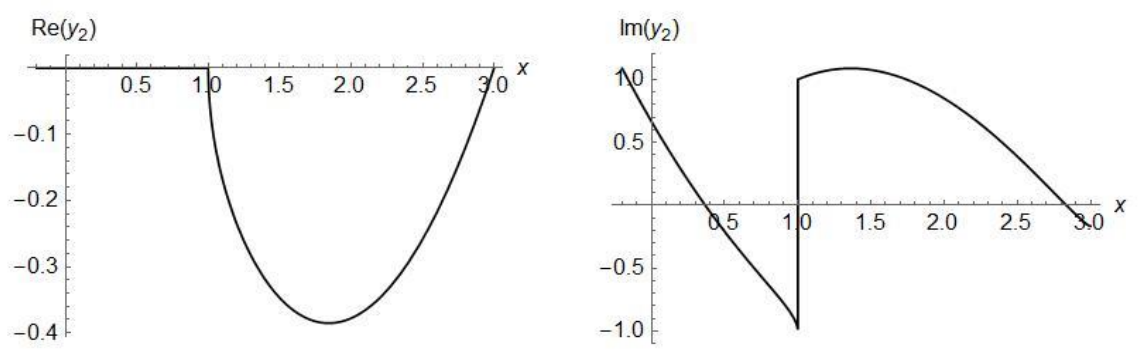

Fig. 7. Graphs of the real and imaginary parts of function $y_{2}(x)$.

\section{The case 9.}

$$
\begin{gathered}
\Lambda=-\beta, \beta=-2(1+4 \gamma), \tau=3 ; \\
y^{\prime \prime}+\frac{2 x-1}{2(x-1) x} y^{\prime}+\frac{\gamma-9 x+7}{4(x-1) x(x-\gamma-1)} y=0 ; \\
y_{1}=\sqrt{x}(\gamma-x+1),
\end{gathered}
$$




$$
y_{2}=\sqrt{x}(\gamma-x+1)\left(\frac{(4 \gamma+1) \tanh ^{-1}\left(\frac{\sqrt{\frac{x-1}{x}}}{\sqrt{\frac{\gamma}{\gamma+1}}}\right)}{\gamma^{3 / 2}(\gamma+1)^{5 / 2}}+\frac{\sqrt{\frac{x-1}{x}}(2 \gamma(\gamma-x+1)+x)}{\gamma(\gamma+1)^{2}(\gamma-x+1)}\right) .
$$

The case 10.

$$
\begin{gathered}
\Lambda=-\beta, \beta=-6(1+2 \gamma), \tau=4 ; \\
y^{\prime \prime}+\frac{2 x-1}{2(x-1) x} y^{\prime}+\frac{2 \gamma-8 x+5}{2(x-1) x(x-\gamma-1)} y=0 ; \\
y_{1}=\sqrt{(1-x) x}(\gamma-x+1), \\
y_{2}=-\sqrt{(1-x) x}(\gamma-x+1)\left(\frac{3(2 \gamma+1) \tanh ^{-1}\left(\frac{\sqrt{\frac{x-1}{x}}}{\sqrt{\frac{\gamma}{\gamma+1}}}\right)}{\gamma^{5 / 2}(\gamma+1)^{5 / 2}}+\frac{-\frac{2}{\gamma^{2}}+\frac{x-1}{\gamma^{2}(\gamma+1)^{2}(\gamma-x+1)}-\frac{2(x-1)}{(\gamma+1)^{2} x}}{\sqrt{\frac{x-1}{x}}}\right) .
\end{gathered}
$$

Functions $y_{1}, y_{2}$ are illustrated by fig. 8,9 (parameters: $\varepsilon=15, \beta=18, \gamma=-2, \Lambda=-18$ ).

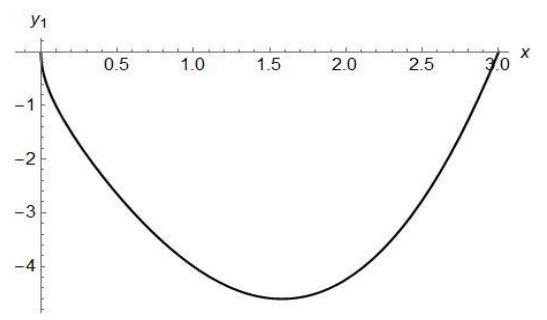

Fig. 8. The graph of the function $y_{1}(x)$.
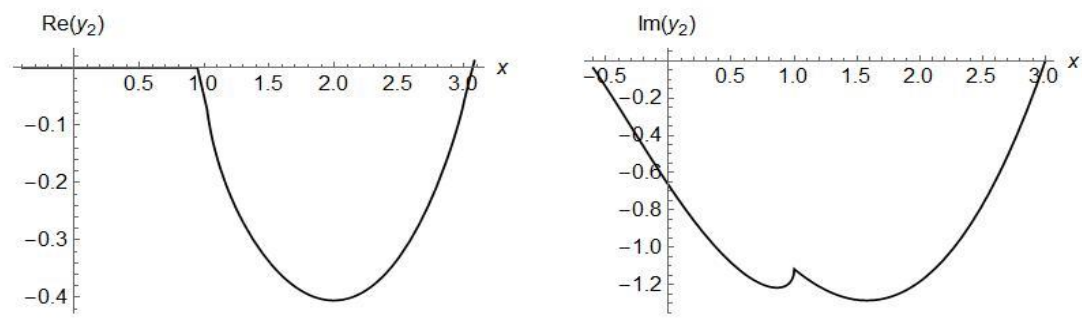

Fig. 9. Graphs of the real and imaginary parts of function $y_{2}(x)$.

The case 11 .

$$
\begin{gathered}
\Lambda=\tau^{2}-3 \tau+2, \beta=-24 \gamma, \tau=5 ; \\
y^{\prime \prime}+\frac{2 x-1}{2(x-1) x} y^{\prime}+\frac{\gamma^{2}+(38-25 x) x-13}{4(x-1) x(x-\gamma-1)(\gamma+x-1)} y=0 ; \\
y_{1}=\sqrt{x}\left((x-1)^{2}-\gamma^{2}\right), \\
y_{2}=\frac{\sqrt{x}\left((x-1)^{2}-\gamma^{2}\right)}{4 \gamma^{7 / 2}} \times\left(\frac{2 \gamma^{3 / 2} \sqrt{\frac{x-1}{x}}\left(-4 \gamma^{4}+\gamma^{2}(x(4 x-9)+4)+x(2 x-3)\right)}{\left(\gamma^{2}-1\right)^{2}\left((x-1)^{2}-\gamma^{2}\right)}-\right.
\end{gathered}
$$




$$
\left.\frac{3(2 \gamma+1) \tan ^{-1}\left(\frac{\sqrt{\gamma} \sqrt{x}}{\sqrt{-\gamma-1} \sqrt{x-1}}\right)}{(-\gamma-1)^{5 / 2}}+\frac{3(1-2 \gamma) \tanh ^{-1}\left(\frac{1}{\sqrt{\frac{\gamma-1}{\gamma}} \sqrt{\frac{x-1}{x}}}\right)}{(\gamma-1)^{5 / 2}}\right) .
$$

The case 12.

$$
\begin{gathered}
\Lambda=\tau^{2}-3 \tau+2, \beta=-32 \gamma, \tau=6 \\
y^{\prime \prime}+\frac{2 x-1}{2(x-1) x} y^{\prime}+\frac{\gamma^{2}+(13-9 x) x-4}{(x-1) x\left((x-1)^{2}-\gamma^{2}\right)} y=0 \\
y_{1}=\sqrt{(1-x) x}\left((x-1)^{2}-\gamma^{2}\right), \\
y_{2}=\frac{\sqrt{(1-x) x}}{4}\left((x-1)^{2}-\gamma^{2}\right) \times\left(\frac{(8 \gamma+5) \tan ^{-1}\left(\frac{\sqrt{\gamma} \sqrt{x}}{\sqrt{-\gamma-1} \sqrt{x-1}}\right)}{(-\gamma-1)^{5 / 2} \gamma^{9 / 2}}-\frac{(8 \gamma-5) \tanh ^{-1}\left(\frac{\sqrt{\gamma} \sqrt{x}}{\sqrt{\gamma-1} \sqrt{x-1}}\right)}{(\gamma-1)^{5 / 2} \gamma^{9 / 2}}\right. \\
\left.-\sqrt{\frac{x-1}{x}}\left(\frac{x}{\gamma^{4}}\left(\frac{1}{(\gamma+1)^{2}(\gamma-x+1)}-\frac{8}{(\gamma-1)^{2}(\gamma+x-1)}-\frac{8}{x-1}\right)-\frac{8}{\left(\gamma^{2}-1\right)^{2}}\right)\right) .
\end{gathered}
$$

Functions $y_{1}$ and $y_{2}$ are illustrated by fig. 10, 11 (parameters $\varepsilon=35, \beta=64, \gamma=-2, \Lambda=20$ ).

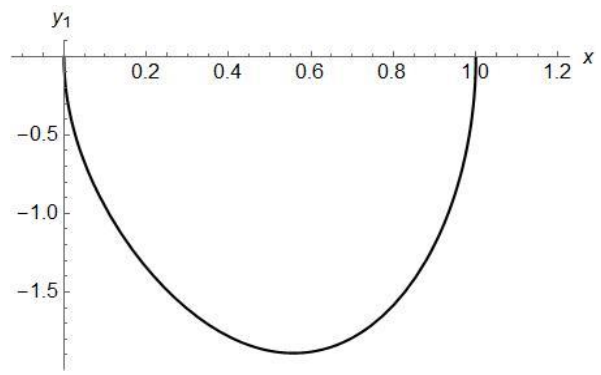

Fig. 10. The graph of the function $y_{1}(x)$.
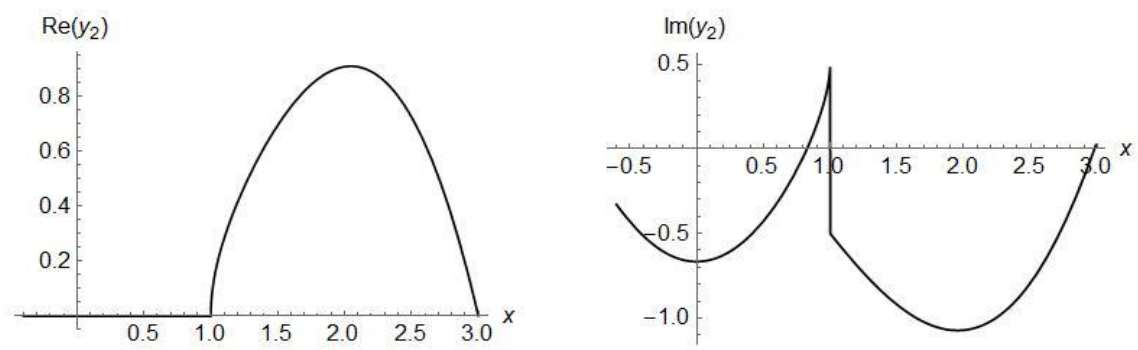

Fig. 11. Graphs of the real and imaginary parts of function $y_{2}(x)$.

The study of the remaining variants II, III and II, I do not lead to new results.

\section{Conclusion}

Because in the quantum-mechanical context, the probability density $|f(x)|^{2}$ must be finite in all points of spherical Riemann space, all above constructed solutions are physically 
interpretable. Though we may conclude that the general approach for producing solutions of ordinary differential equations in elementary functions is operable, however it is effective only for special values of all involved parameters.

\section{References}

1. N.A. Lukashevich, A.V. Chichurin, Differential equations of the first order (BSU, Minsk, 1999) (in Russian)

2. A.V. Chichurin, Chazy equation and linear equations of the Fuchs class (RUDN, Moscow, 2003) (in Russian)

3. A. V. Chichurin, Nonlinear Oscillations 6, 277 (2003)

4. N. A Lukashevich, Differ. Equ. 35, 1384 (1999)

5. A.V. Chichurin, G.P. Stepaniuk, Bulletin of Taras Shevchenko National University of Kyiv, Series: Physics and Mathematics, 31, 29 (2014)

6. A. Chichurin, in: Recent Developments in Mathematics and Informatics, Vol. I. Contemporary Mathematics and Computer Science (KUL, Lublin, 2016)

7. V.V. Kisel, E.M. Ovsiyuk, O.V. Veko, Y.A. Voynova, V. Balan, V.M. Red'kov, Elementary Particles with Internal Structure in External Fields. Vol I. General Theory; Vol II. Physical Problems (Nova Science Publishers Inc., New York, 2018)

8. A.V. Chichurin, E.M. Ovsiyuk, V.M. Red'kov, Computers and Mathematics with Applications. 75. 1550 (2018) 